Purdue University

Purdue e-Pubs

2005

\title{
Dynamic Response Optimization of Piezoelectrically Excited Thin Resonant Beams
}

S. Basak

A. Raman

Purdue University

S V. Garimella

Purdue University, sureshg@purdue.edu

Follow this and additional works at: http://docs.lib.purdue.edu/coolingpubs

Basak, S.; Raman, A.; and Garimella, S V., "Dynamic Response Optimization of Piezoelectrically Excited Thin Resonant Beams" (2005). CTRC Research Publications. Paper 290.

http://dx.doi.org/10.1115/1.1857921

This document has been made available through Purdue e-Pubs, a service of the Purdue University Libraries. Please contact epubs@purdue.edu for additional information. 


\section{Dynamic Response Optimization of Piezoelectrically Excited Thin Resonant Beams}

\author{
Arvind Raman* \\ e-mail: raman@ecn.purdue.edu
}

\section{Suresh V. Garimella \\ School of Mechanical Engineering, \\ Purdue University, \\ West Lafayette, Indiana 47907-2088}

Piezoelectrically excited, resonant, elastic beams find wide use as piezoelectric fans, optical choppers, MEMS sensors, and piezoelectric motors. The devices consist of either one piezoelectric ceramic patch (piezopatch) bonded on one side (asymmetric configuration), or of two oppositely poled patches placed symmetrically on either side of a thin, flexible elastic beam (symmetric configuration). Field equations of the coupled structure governing the coupled longitudinal and bending motions of the resonator are derived using linear constitutive equations, slender beam approximations, and Hamilton's principle. Analytical solutions are found to the coupled eigenvalue problem. Eigenvalues and eigenfunctions for the short-circuited and open-circuited configurations are predicted analytically and are found to be in excellent agreement with results from threedimensional finite element simulations. Electromechanical coupling factors (EMCF) are computed using the analytical and finite element model and optimal resonator geometries are identified for maximal EMCF. The EMCF predictions are also compared with experiments for an asymmetrically configured resonator. The analytical solution provides a convenient tool for the optimal design of such devices. [DOI: 10.1115/1.1857921]

\section{Introduction}

Flexural piezoelectric resonators are solid-state devices consisting of piezoelectric elements bonded to an underlying elastic structure. A properly tuned harmonic voltage input excites piezoelectrically a flexural resonance in the structure. Such resonators are used as actuators and sensors for a wide variety of applications including piezoelectric fans, optical beam choppers, ultrasonic choppers, MEMS density sensors, resonating viscometers, ultrasonic motors, automatic tooth brushes, and dentistry tools. In particular, piezoelectric fans utilize this flexural vibration to induce vortices and streaming flow for electronics cooling. Figure 1 shows a single-patch commercial piezoelectric fan which is made of mylar and operates at a nominal bending resonance of $60 \mathrm{~Hz}$.

These fans were first discussed by Toda [1]. Piezoelectric fans have gained importance recently for cooling applications in modern portable electronics such as laptops and cellphones and automobile multimedia boxes. The use of piezoelectric fans as very compact, low power, noiseless air cooling devices has been studied recently by Yoo et al. [2] and Campbell et al. [3]. For the design of flexural resonators in piezoelectric fans and other applications, little seems to be known about an optimal actuator-beam configuration. Indeed the optimization objective may vary among these devices. However for piezoelectric fans a clear objective is to maximize the output mechanical energy for a given available electrical energy input. This leads to faster streaming and thus to effective cooling. To achieve this, the so-called electromechanical coupling factor (EMCF) needs to be maximized for each chosen resonant mode. Likewise, maximal EMCF is one of the natural optimization objectives for other devices such as optical choppers and resonant motors.

The literature on piezoelectric beam systems can be divided broadly into that dealing with static actuation $([4-7])$ and that focused on dynamic analysis $([8-19])$ of infinite and finite beams. Gibbs and Fuller [8], Pan and Hansen [9], Brennan et al. [10], and more recently, Wolf [11] investigated the dynamics of structures consisting of a finite length piezopatch attached to an infinite

\footnotetext{
*Corresponding author

Contributed by the Technical Committee on Vibration and Sound for publication in the JOURNAL OF VIBRATION AND ACOUSTICS. Manuscript received May 9, 2003 final revision March 29, 2004. Review conducted by J. Main.
}

beam. Gibbs and Fuller [8] and Brennan et al. [10] modeled the flexural and longitudinal waves generated in the beam by the piezopatch. Pan and Hansen [9] modeled piezoelectric actuation as an equivalent point force located at the center of the actuator. Wolf [11] derived the equations of motion using variational principle. The EMCF dependence on the patch thickness was investigated analytically and using finite element methods. However in most applications outlined earlier, both the patch and the underlying beam are of finite dimensions. Flexural and longitudinal waves generated by the piezoelectric excitation reflect off the beam boundaries requiring a separate, detailed analysis of the finite beam.

Dynamics of finite beams were analyzed in [12-19]. Pan et al. [12] and Rivory et al. [13] presented a dynamical model of a finite beam with symmetrically placed piezopatches. They did not compute the EMCF and neglected the added mass and stiffness effects of the piezopatches. Kim and Jones [14] studied the statics and dynamics of symmetric and asymmetric configurations. However, their dynamic analysis was restricted to cases where the piezopatch covered entirely the underlying beam. As will be demonstrated in this paper, the problem is significantly complicated when the patch is shorter than the beam. Rontong et al. [15] studied the bending and extension of a cantilever with symmetrically placed piezopatches using the Rayleigh-Ritz approach. They also assumed a constrained layer between the beam and the patches. Lobontiu et al. [16] used a lumped parameter approach to predict the resonant tip deflection. The mass, damping, stiffness, and forcing terms were derived as parameters of the piezoelectrically actuated system. Barboni et al. [17] analyzed a beam covered partially by two piezopatches using the kinematic assumptions of Crawley and de Luis [4]. However, the additional mass and stiffness of the piezopatches are not considered. Optimal location and geometries of the piezopatches were investigated to improve the controllability of the structure. Wang and Wang [18] modeled the effects of the piezopatches as internal bending moments, neglecting the effects of beam deflection on the piezopatch. Bürmann et al. [19] analyzed a partially covered, symmetrically configured, double-patch finite beam.

Furthermore, [5-10] and [12-18] assume a constant electric field across the patch thickness in conjunction with Bernoulli- 


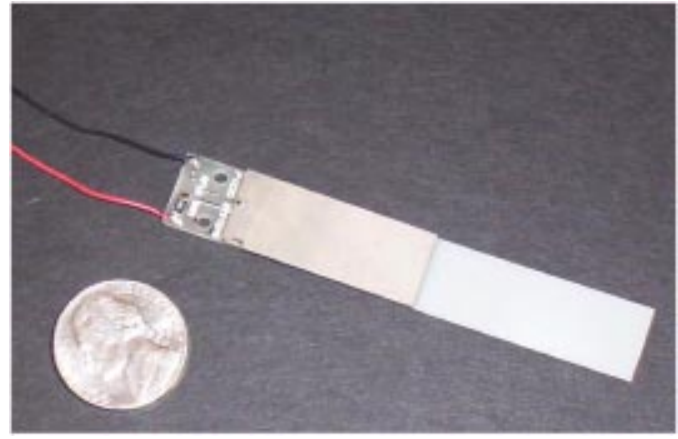

Fig. 1 A photograph of a single-patch (asymmetric configuration) commercial fan from Piezo Systems, Inc.

Euler strain distribution in the patches. However these two assumptions together violate Gauss' law, which requires a vanishing charge in the electrically insulated dielectric.

This paper follows the developments of [19] and extends the analysis to the more complicated and practically relevant case of finite beams in both symmetric and asymmetric configurations. Modeling the asymmetric configuration is more complicated than the symmetric configuration because both bending and extensional motions are excited simultaneously. Moreover, the neutral axis jumps discontinuously across the ends of the actuator. The main contributions of this paper include: (i) analytical modeling of the coupled dynamics of a piezopatch attached to a thin, finite elastic beam in the symmetric and asymmetric configurations; (ii) derivation of an analytical solution of the short-circuited and open-circuited eigenvalue problem; (iii) predictions of optimal configurations based on EMCF; (iv) verification of the results using finite element predictions; and (v) comparison with experimental results obtained with asymmetrically configured resonators.

\section{Electromechanical Modeling and Analysis}

2.1 Kinematics. A schematic of the key kinematic variables used in the analysis is presented in Fig. 2. Unless otherwise noted, the subsequent development is common to both the symmetric and asymmetric configurations. The composite elastic beam is divided into three regions: Region 1 extends from the clamp up to the start of the patch; region 2 is the composite piezopatch-elastic beam section; and region 3 extends from the end of the patch to the free end of the elastic beam. The structure in each region is modeled separately as a thin Bernoulli-Euler beam. The model is assumed to be free of all kind of losses. The piezopatches are assumed to be bonded perfectly to the elastic beam through an infinitesimal bonding layer. The neutral axis in region 1 and region 3 lies along the centroidal axis of the beam cross section, while its location in region 2 depends on its configuration. In the symmetric configuration [Fig. 2(b)], the neutral axis in region 2 is identical to that in region 1 and region 3 . For the asymmetric configuration, however, the neutral axis in region 2 is located a distance $e$ below the patch-beam interface [Fig. 2(a)], where [11]

$$
e=\frac{t_{b}}{2}\left[\frac{\left(\frac{E_{b}}{E_{p}}-\left(\frac{t_{p}}{t_{b}}\right)^{2}\right)}{\frac{E_{b}}{E_{p}}+\left(\frac{t_{p}}{t_{b}}\right)}\right]
$$

The beam is made of a linearly elastic, isotropic material while the piezopatch is planar isotropic [20]. Planar isotropy is usually assumed for initially strongly polarized piezoelectric materials. For thin beams undergoing small amplitude oscillations and crosssectional rotations, the Bernoulli-Euler beam model is applied separately to each region. Accordingly the longitudinal normal strain $\varepsilon_{1}$ is approximated by

$$
\varepsilon_{1}=u^{\prime}-x_{3} w^{\prime \prime}
$$

where ' denotes the derivative with respect to $x_{1}$, and $x_{3}$ is mea-

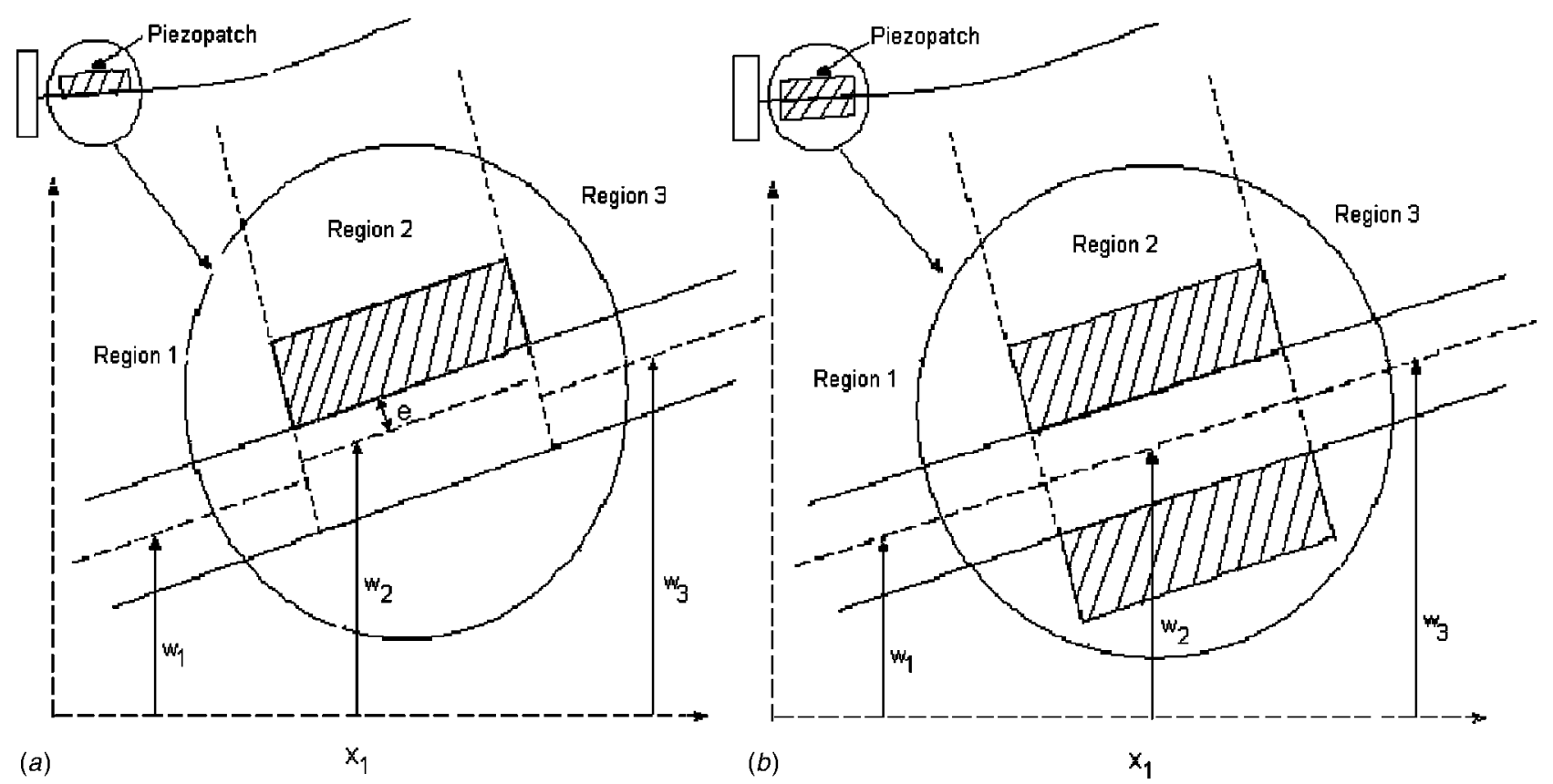

Fig. 2 Schematic diagram displaying the kinematic quantities of the (a) asymmetrically and (b) symmetrically configured resonators. Note that in (a) the neutral axis jumps at the interface between regions 1, 2 and 3. 
sured from the neutral axis for each individual region. In Eq. (2), $u$ and $w$ stand for the longitudinal and transverse displacements, respectively.

2.2 Electric Enthalpy. The electrical enthalpy [20] of the piezopatch is now derived under the assumption of thin beam theory. The axis of polarization of each patch is the $x_{3}$ axis. The planar, isotropic piezoelectric constitutive equations are [20]

$$
\mathbf{T}=\mathbf{c S}-\mathbf{e E}, \quad \mathbf{D}=\mathbf{e}^{\mathrm{T}} \mathbf{S}+\epsilon \mathbf{E}
$$

where T, S, E, and $\mathbf{D}$ denote, respectively, stress, strain, electric field, and electric displacement vector while c, e, $\epsilon$ contain, respectively, the components of linear moduli, the piezoelectric coefficients, and the dielectric constants.

Assuming a state of uniaxial stress $\sigma_{1}$ and uniaxial electric field $E_{3}$ and $D_{3}$, the linear constitutive equations [20] for piezopatches reduce to [11]

$$
\sigma_{1}=c_{11} \varepsilon_{1}-e_{31} E_{3}, \quad D_{3}=e_{31} \varepsilon_{1}+\epsilon_{33} E_{3}
$$

The constants in the constitutive equations are given by

$$
\epsilon_{33}=K_{33} \epsilon_{0}, \quad c_{11}=E_{p}, \quad e_{31}=E_{p} d_{31}
$$

where $K_{33}, \varepsilon_{0}, E_{p}$, and $d_{31}$ denote respectively the relative dielectric constant, the permittivity of free space, the Young's modulus of the piezoelectric actuator, and the piezoelectric strain coefficient.

The absence of free charges inside the actuator requires $\boldsymbol{\nabla}$.D $=0[11]$. For the one-dimensional case under consideration, this further reduces to

$$
D_{3,3}=0
$$

where the index 3 denotes the derivative of $D_{3}$ with respect to $x_{3}$. In addition, the electric potential $\Phi$ is introduced such that $\mathbf{E}=-\nabla \Phi$. For thin piezopatches, this reduces to

$$
E_{3}=-\phi_{, 3} \text {. }
$$

Substitution of Eqs. (2) and (5) into Eq. (3) yields

$$
\varepsilon_{33} E_{3,3}-e_{31} w^{\prime \prime}=0 .
$$

Equations (6) and (7) lead to the conclusion that the electric potential function must be quadratic in $x_{3}$

$$
\Phi\left(x_{3}\right)=\phi_{0}+\phi_{1} x_{3}+\phi_{2} x_{3}^{2}
$$

Substitution of Eqs. (6) and (8) into Eq. (7) leads to

$$
\phi_{2}=-\frac{1}{2} \frac{e_{31}}{\varepsilon_{33}} w^{\prime \prime} .
$$

Further, using the boundary condition

$$
\phi\left(e+t_{p}\right)-\phi(e)=\Delta \phi
$$

where $\Delta \phi$ is the potential difference between the bottom and the top of electrodes, together with Eq. (8) leads to

$$
\phi_{1}=\frac{\Delta \phi}{t_{p}}+\frac{1}{2} \frac{e_{31}}{\varepsilon_{33}}\left(2 e+t_{p}\right) w^{\prime \prime}
$$

Equations (9) and (10) together with Eq. (8) then lead to the approximate form of the electric field in the piezopatch

$$
E_{3}\left(x_{1}, x_{3}, t\right)=-\frac{2 \varepsilon_{33} \Delta \phi+e_{31} h_{p}\left(2 e+t_{p}-2 x_{3}\right) w^{\prime \prime}}{2 \varepsilon_{33} t_{p}}
$$

It may be noted that the electric field $E_{3}$ depends linearly on the potential difference $\Delta \phi$ and the curvature $w^{\prime \prime}$. Because $\sigma_{1}$ and $D_{3}$ are related to the internal energy of the piezoelectric actuator [20] through

$$
\sigma_{1}=\frac{\partial H}{\partial \varepsilon_{1}}, \quad D_{3}=-\frac{\partial H}{\partial E_{3}}
$$

the total enthalpy of the piezopatch is given by [20]

$$
H=\frac{1}{2} c_{11} \varepsilon_{1}^{2}-e_{31} E_{3} \varepsilon_{1}-\frac{1}{2} \varepsilon_{33} E_{3}^{2}
$$

Substitution of the approximations of strain (2) and electric field (11) into Eq. (13) leads to

$$
\begin{aligned}
H= & \frac{1}{2} E_{p} u^{\prime 2}+\left(a_{2} x_{3}^{2}+a_{3}\right) w^{\prime \prime 2}+a_{4} w^{\prime \prime}+a_{5}+\left(a_{6}+a_{7} x_{3}\right) u^{\prime} w^{\prime \prime} \\
& +a_{8} u^{\prime}
\end{aligned}
$$

where the constants in Eq. (14) are given by

$$
\begin{gathered}
a_{2}=\frac{1}{2}\left(c_{11}+\frac{e_{31}^{2}}{\varepsilon_{33}}\right), \quad a_{3}=\frac{-e_{31}^{2}\left(2 e+t_{p}\right)^{2}}{8 \varepsilon_{33}}, \\
a_{4}=-\frac{e_{31}\left(2 e+t_{p}\right) \Delta \phi}{2 t_{p}}, \\
a_{5}=\frac{-\varepsilon_{33}(\Delta \phi)^{2}}{2\left(h_{p}\right)^{2}}, \quad a_{6}=\frac{e_{31}^{2}}{2 \varepsilon_{33}}\left(2 e+t_{p}\right), \\
a_{7}=-\left(E_{p}+\frac{e_{31}^{2}}{\varepsilon_{33}}\right), \quad a_{8}=e_{31} \frac{\Delta \phi}{t_{p}}
\end{gathered}
$$

The potential difference $\Delta \phi$ is prescribed as an electrical boundary condition, that is, the electrodes of the actuator are either short-circuited (SC)

$$
\Delta \phi=0
$$

or open-circuited (OC) and the charges on the electrodes are generated naturally by bending. Upon assumption that the top electrode is initially charge-free $\int_{L 1}^{L 2} D_{3} d x_{1}=0$ and using Eqs. (3) and (11) the open-circuited boundary condition may be obtained

$$
\Delta \phi=\frac{e_{31} t_{p}}{(L 2-L 1) \epsilon_{33}}\left[\left.u\right|_{L 1} ^{L 2}-\left.\frac{\left(t_{p}+2 e\right)}{2} w^{\prime}\right|_{L 1} ^{L 2}\right]
$$

Equation (17) relates the charge developed to the difference in the displacement fields at the beginning and the end of the electrode.

2.3 Equations of Motion and Boundary Conditions. Upon neglect of the rotational inertia of the beam and the patch the Lagrangian for the resonator can be formulated as

$$
L=L_{b}+L_{p}, \quad L_{b}=T_{b}-U_{b}, \quad L_{p}=T_{p}-U_{p}
$$

where

$$
\begin{gathered}
T_{b}=\int_{V_{b}} \frac{\rho_{b}}{2} \dot{u}^{2} d V_{b}+\int_{V_{b}} \frac{\rho_{b}}{2} \dot{w}^{2} d V_{b}, \\
T_{p}=\int_{V_{p}} \frac{\rho_{p}}{2} \dot{u}^{2} d V_{p}+\int_{V_{p}} \frac{\rho_{p}}{2} \dot{w}^{2} d V_{p}, \\
U_{b}=\int_{V_{b}} \frac{1}{2} E_{b}\left(u^{\prime}-x_{3} w^{\prime \prime}\right)^{2} d V_{b}, \quad U_{p}=\int_{V_{p}} H d V_{p}
\end{gathered}
$$

and $\rho_{b}, \rho_{p}, V_{b}, V_{p}$, and $E_{b}$ denote, respectively, the mass density of the beam and the piezopatch, the volume of the beam and the piezopatch, and the Young's modulus of the beam. $H$ is the enthalpy of the piezoelectric actuator derived in the previous section. $u$ and $w$ are the longitudinal and transverse displacements, respectively. The equations of motion of the system are derived by setting the first variation of action to zero [11], such that

$$
\delta \int_{t_{1}}^{t_{2}} \sum_{i=1}^{3} L_{i} d t=0
$$

where $L_{i}$ denotes the Lagrangian that is defined piecewise for region 1 , region 2 and region 3 , respectively.

The above formulation is valid for both the asymmetric (singlepatch) and symmetric (two-patch) configurations. The patches on 
Table 1 Material properties used in this paper. A polarized ferroelectric ceramic model with $x_{3}$ as the poling direction was used in the analysis. Piezoelectric material properties are obtained from Piezo Systems, Inc. catalog 3.

\begin{tabular}{lll}
\hline \hline & \multicolumn{1}{c}{ Physical Quantity } & \multicolumn{1}{c}{ Value } \\
\hline$E_{b}$ & Young's modulus of the beam (brass) & $10 \times 10^{10} \mathrm{~N} / \mathrm{m}^{2}$ \\
$E_{p}$ & Young's modulus of the actuator (PSI-5H-S4-ENH) & $6.2 \times 10^{10} \mathrm{~N} / \mathrm{m}^{2}$ \\
$\rho_{b}$ & Density of the beam (brass) & $8400 \mathrm{~kg} / \mathrm{m}^{3}$ \\
$\rho_{p}$ & Density of the actuator (PSI-5H-S4-ENH) & $7800 \mathrm{~kg} / \mathrm{m}^{3}$ \\
$t_{b}$ & Thickness of the beam & $0.1016 \mathrm{~mm}$ \\
$t_{p}$ & Thickness of the actuator & $0.05 t_{b}-1.5 t_{b}$ \\
$b$ & Width of beam and actuator & (varied in steps of $\left.0.05 t_{b}\right)$ \\
$L 1$ & Length of region 1 & $8.89 \mathrm{~mm}$ \\
$L 2$ & Length of patch & $0.5 \mathrm{~mm}$ \\
& Overall length of the structure $L 3$ & (varied in steps of $0.03 L 3$ ) \\
$L_{33}$ & Relative dielectric constant & $27.424 \mathrm{~mm}$ \\
$\epsilon_{0}$ & Permittivity of free space & 3800 \\
$d_{31}$ & Piezoelectric strain coefficient & $8.8542 \times 10^{-12} \mathrm{C} / \mathrm{V} \mathrm{m}$ \\
\hline \hline
\end{tabular}

either side of the symmetrically configured beam are either driven in phase exciting longitudinal motions, or out of phase inducing pure flexure [4]. For either type of motion, only the longitudinal or the transverse vibration terms need to be retained in Eq. (18).

Application of Hamilton's principle for the asymmetric configuration results in two equations of motion for each region, one corresponding to the longitudinal motion and the other corresponding to the transverse motion. In all, there are 18 boundary conditions-four each associated with the transverse motion in each region and two each for the longitudinal motion of each region. The SC and OC electrical boundary conditions are implemented through terms in the equations of motion involving $\Delta \phi$. $\Delta \phi$ in turn is prescribed by the electrical boundary conditions [Eqs. (16) and (17)].

The equations of motion in the three regions are given as follows:

Region 1

$$
-\rho_{b} A_{b} \ddot{u}_{1}+E_{b} A_{b} u_{1}^{\prime \prime}=0, \quad \rho_{b} A_{b} \ddot{w}_{1}+E_{b} I_{b} w_{1}^{\prime \prime \prime \prime}=0
$$

Region 2

$$
\begin{gathered}
-\left(\rho_{b} A_{b}+\rho_{p} A_{p}\right) \ddot{u}_{2}+\left(E_{b} A_{b}+E_{p} A_{b}\right) u_{2}^{\prime \prime}=0 \\
-\left(\rho_{b} A_{b}+\rho_{p} A_{p}\right) \ddot{w}_{2}-\left(2 a_{2} I_{p e}+2 a_{3} A_{p}+E_{b} I_{b e}\right) w_{2}^{\prime \prime \prime \prime}=0
\end{gathered}
$$

Region 3

$$
-\rho_{b} A_{b} \ddot{u}_{3}+E_{b} A_{b} u_{3}^{\prime \prime}=0, \quad \rho_{b} A_{b} \ddot{w}_{3}+E_{b} I_{b} w_{3}^{\prime \prime \prime \prime}=0
$$

The geometric boundary conditions are given by

$$
\begin{gathered}
u_{1}(0, t)=0, \quad w_{1}(0, t)=0, \quad w_{1}^{\prime}(0, t)=0 \\
u_{1}(L 1, t)=u_{2}(L 1, t), \quad w_{1}(L 1, t)=w_{2}(L 1, t), \\
w_{1}^{\prime}(L 1, t)=w_{2}^{\prime}(L 1, t) \\
u_{2}(L 2, t)=u_{3}(L 2, t), \quad w_{2}(L 2, t)=w_{3}(L 2, t), \\
w_{2}^{\prime}(L 2, t)=w_{3}^{\prime}(L 2, t) .
\end{gathered}
$$

And the natural boundary conditions are

$$
\begin{gathered}
\left(A_{p} E_{p}+E_{b} A_{b}\right) u_{2}^{\prime}(L 1, t)+a_{8} A_{p}=E_{b} A_{b} u_{1}^{\prime}(L 1, t) \\
\left(2 a_{2} I_{p e}+2 a_{3} A_{p}+E_{b} I_{b e}\right) w_{2}^{\prime \prime}(L 1, t)+a_{4} A_{p}=E_{b} I_{b} w_{1}^{\prime \prime}(L 1, t) \\
\left(2 a_{2} I_{p e}+2 a_{3} A_{p}+E_{b} I_{b e}\right) w_{2}^{\prime \prime \prime}(L 1, t)=E_{b} I_{b} w_{1}^{\prime \prime \prime}(L 1, t) \\
\left(A_{p} E_{p}+E_{b} A_{b}\right) u_{2}^{\prime}(L 2, t)+a_{8} A_{p}=E_{b} A_{b} u_{3}^{\prime}(L 2, t) \\
\left(2 a_{2} I_{p e}+2 a_{3} A_{p}+E_{b} I_{b e}\right) w_{2}^{\prime \prime}(L 2, t)-a_{4} A_{p}=E_{b} I_{b} w_{3}^{\prime \prime}(L 2, t)
\end{gathered}
$$

$$
\begin{gathered}
\left(2 a_{2} I_{p e}+2 a_{3} A_{p}+E_{b} I_{b e}\right) w_{2}^{\prime \prime \prime}(L 2, t)=E_{b} I_{b} w_{3}^{\prime \prime \prime}(L 2, t) \\
u_{3}^{\prime}(L 3, t)=0, \quad w_{3}^{\prime \prime}(L 3, t)=0, \quad w_{3}^{\prime \prime \prime}(L 3, t)=0 .
\end{gathered}
$$

Equations (20)-(22) govern the dynamics of the asymmetric configuration. The longitudinal and transverse motions in the equations of motion are decoupled in each region. The coupling arises, however, in the boundary conditions through the terms $a_{4}$ and $a_{8}$ in Eqs. $(23 a),(23 b),(23 d)$ and $(23 e)$. The corresponding equations for the symmetric configuration can be easily derived as a special case from the above equations by setting the transverse components to zero when the patches are driven in phase and by setting the longitudinal components to zero when the patches are driven out of phase.

2.4 Solution Procedure. Introduction of a time-separable solution

$$
\begin{gathered}
u_{i}\left(x_{1}, t\right)=\mathfrak{R}\left[U_{i}\left(x_{1}\right) e^{j \Omega t}\right], \\
w_{i}\left(x_{1}, t\right)=\mathfrak{R}\left[W_{i}\left(x_{1}\right) e^{j \Omega t}\right] \quad i=1,2,3
\end{gathered}
$$

for the displacement fields in the three regions results in the following general solution

$$
\begin{aligned}
& W_{i}\left(x_{1}\right)= C_{i, 3} \sin \left(\delta_{i} x\right)+C_{i, 4} \cos \left(\delta_{i} x\right)+C_{i, 5} \sinh \left(\delta_{i} x\right) \\
&+C_{i, 6} \cosh \left(\delta_{i} x\right) \\
& U_{i}\left(x_{1}\right)=C_{i, 1} \sin \beta_{i} x+C_{i, 2} \cos \beta_{i} x \quad i=1,2,3
\end{aligned}
$$

Table 2 The natural frequencies of the analytical and the finite element model for the asymmetric configuration. The finite element model is used as reference when computing the error.

\begin{tabular}{cccc}
\hline \hline Mode & $L 2=7 \mathrm{~mm}$ & $L 2=12 \mathrm{~mm}$ & $L 2=17 \mathrm{~mm}$ \\
\hline First SC anal. & $117.60 \mathrm{~Hz}$ & $161.68 \mathrm{~Hz}$ & $179.50 \mathrm{~Hz}$ \\
First SC FEM & $117.81 \mathrm{~Hz}$ & $158.68 \mathrm{~Hz}$ & $172.98 \mathrm{~Hz}$ \\
Error & $-0.18 \%$ & $1.89 \%$ & $3.77 \%$ \\
& & & \\
First OC anal. & $118.13 \mathrm{~Hz}$ & $163.25 \mathrm{~Hz}$ & $181.82 \mathrm{~Hz}$ \\
First OC FEM & $118.38 \mathrm{~Hz}$ & $160.52 \mathrm{~Hz}$ & $175.67 \mathrm{~Hz}$ \\
Error & $-0.21 \%$ & $1.70 \%$ & $3.50 \%$ \\
& & & \\
Second SC anal. & $595.07 \mathrm{~Hz}$ & $533.32 \mathrm{~Hz}$ & $652.87 \mathrm{~Hz}$ \\
Second SC FEM & $595.29 \mathrm{~Hz}$ & $535.77 \mathrm{~Hz}$ & $649.99 \mathrm{~Hz}$ \\
Error & $-0.04 \%$ & $-0.46 \%$ & $0.44 \%$ \\
Second OC anal. & $598.11 \mathrm{~Hz}$ & $534.30 \mathrm{~Hz}$ & $653.32 \mathrm{~Hz}$ \\
Second OC FEM & $598.45 \mathrm{~Hz}$ & $536.68 \mathrm{~Hz}$ & $651.25 \mathrm{~Hz}$ \\
Error & $-0.06 \%$ & $-0.44 \%$ & $0.32 \%$ \\
\hline \hline
\end{tabular}



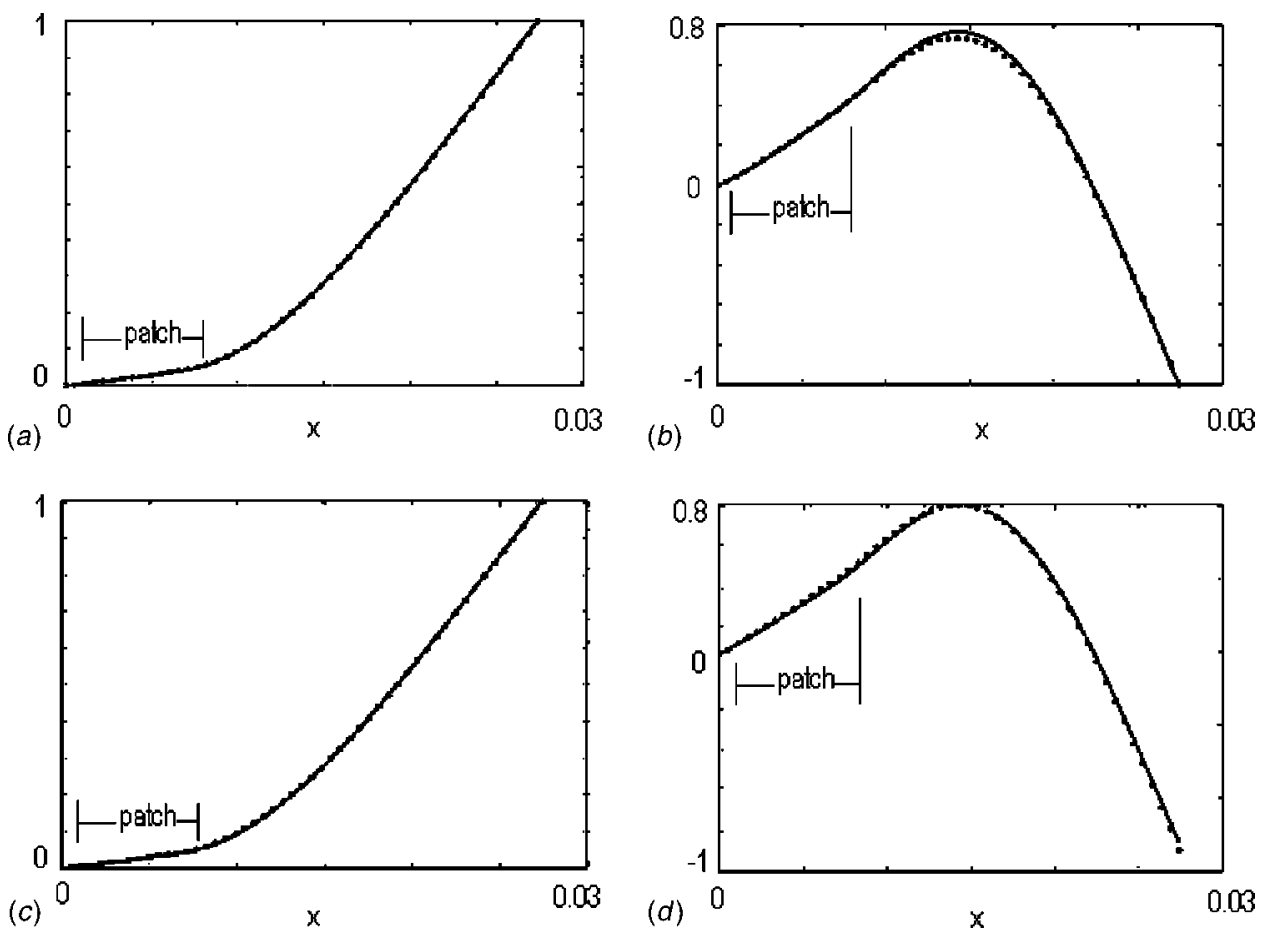

Fig. 3 Transverse vibration component of the first and second bending dominated modes of the asymmetrically configured resonator: (a) SC first Mode, (b) SC second Mode, (c) OC first Mode, and (d) OC second Mode. Solid black line: analytical; stars: finite element predictions.

where

$$
\begin{gathered}
\beta_{1,3}=\left(\frac{\rho_{b} \omega^{2}}{E_{b}}\right)^{1 / 2}, \quad \delta_{1,3}=\left(\frac{\rho_{b} A_{b} \omega^{2}}{E_{b} I_{b}}\right)^{1 / 4}, \\
\beta_{2}=\left(\frac{\left(\rho_{b} A_{b}+\rho_{p} A_{p}\right) \omega^{2}}{E_{b} A_{b}+E_{p} A_{p}}\right)^{1 / 2}, \quad \delta_{2}=\left(\frac{\left(\rho_{b} A_{b}+\rho_{p} A_{p}\right) \omega^{2}}{2 a_{2} I_{p}+2 a_{3} A_{p}+E_{b} I_{b e}}\right)^{1 / 4} .
\end{gathered}
$$

On implementation of the geometric boundary conditions at $x$ $=0$, the constants $C_{1,2}$ vanish and $C_{1,5}=-C_{1,3}$ and $C_{1,6}=$ $-C_{1,4}$. The remaining 15 boundary constants are used to construct a $15 \times 15$ matrix containing the unknown modal frequencies. Vanishing values of the determinant of this matrix yield the natural frequencies and mode shapes of the structure. MAPLE 8 [21] is used to compute the zeros of the determinant. This leads to the exact eigenvalues and eigenfunctions of the structure. For the symmetric configuration this matrix will be only $5 \times 5$ for a purely longitudinal resonator and $10 \times 10$ for a purely flexural resonator.

2.5 Electromechanical Coupling Factor (EMCF). The EMCF is a measure of the obtainable mechanical work in a quasistatic deformation cycle of the piezoceramic. This is conveniently expressed in terms of the SC and the OC strain energies as [11]

$$
\mathrm{EMCF}=\left(\frac{U_{\mathrm{OC}}-U_{\mathrm{SC}}}{U_{\mathrm{OC}}}\right)^{1 / 2}=\left(\frac{U_{\text {conv }}}{U_{\mathrm{SC}}^{p}+U_{\text {conv }}+U_{b}}\right)^{1 / 2},
$$

where $U_{\mathrm{OC}}$ is the total energy stored in the structure when the patch is open-circuited (OC), $U_{\mathrm{SC}}$ is the total energy stored in the structure when the patch is short-circuited (SC), $U_{\text {conv }}$ is the convertible energy, $U_{\mathrm{SC}}^{p}$ is the energy stored in the patch alone with short-circuited electrodes, and $U_{b}$ is the total strain energy stored in the beam.
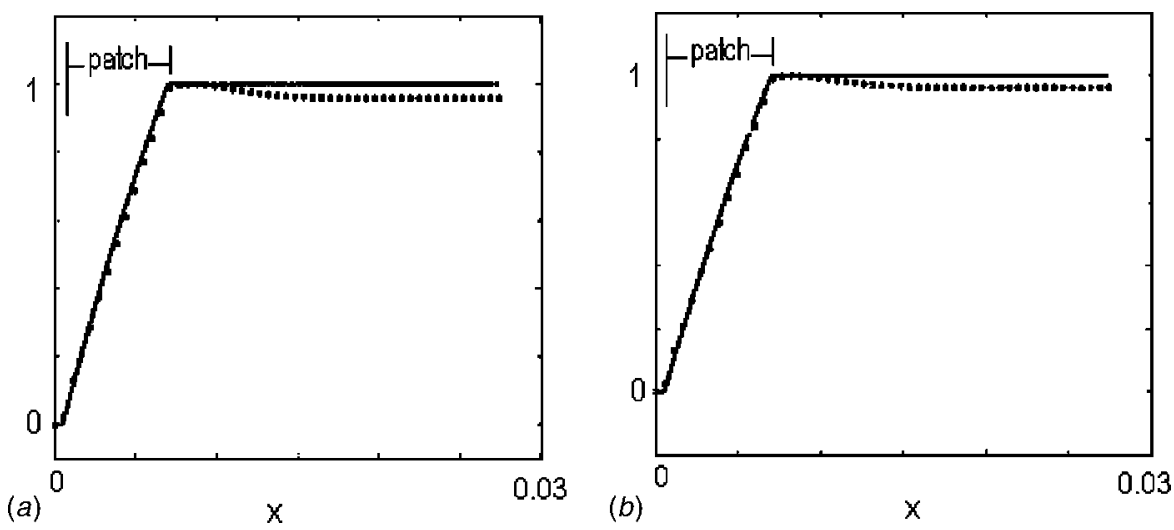

Fig. 4 Longitudinal displacement component of the first bending dominated mode of the asymmetrically configured resonator: (a) SC mode, and (b) OC mode. Solid line: analytical; stars: finite element. 

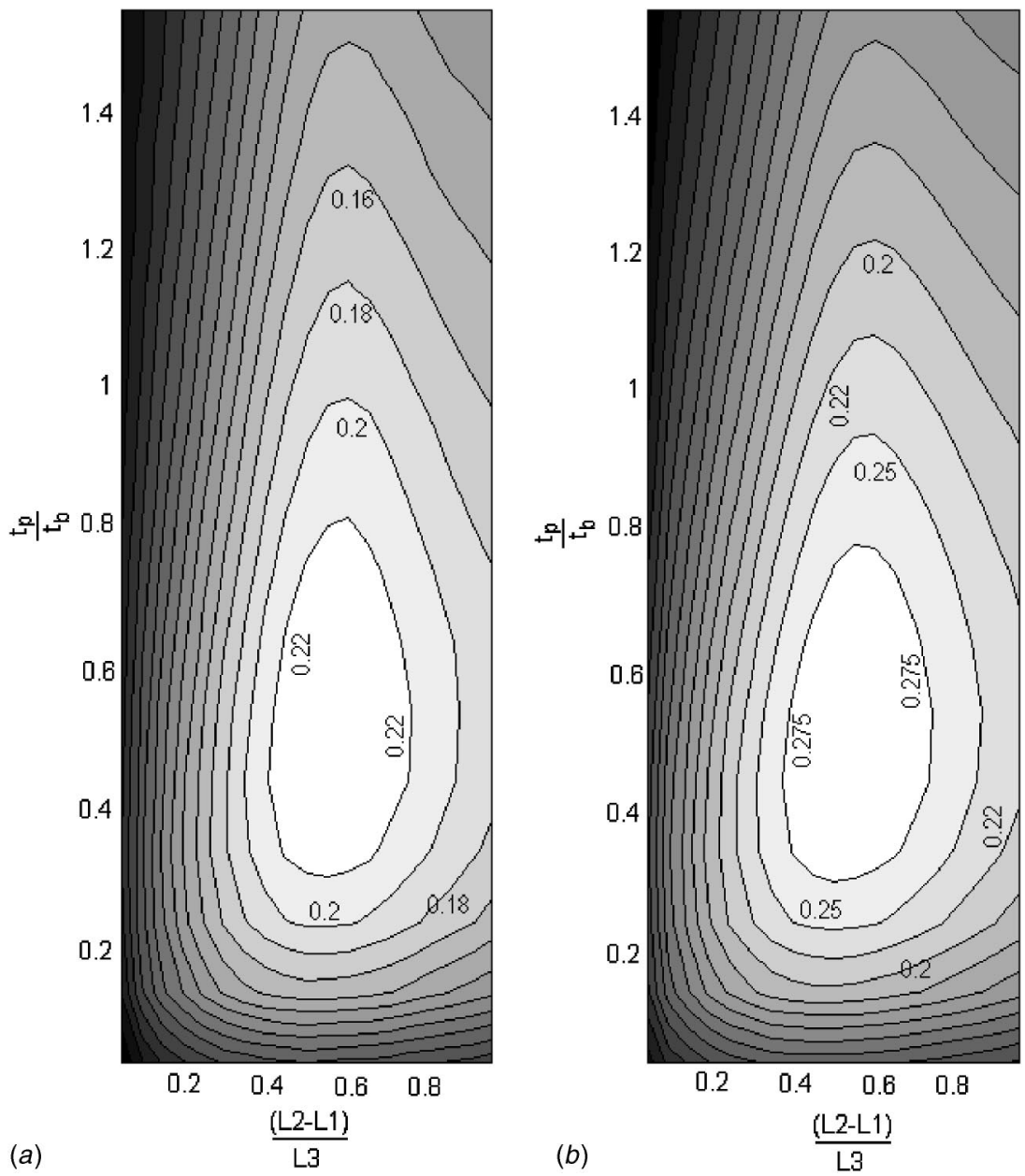

Fig. 5 EMCF variation of the first bending mode of an asymmetric resonator with patch-to-beam length ratio $(L 2-L 1) / L 3$ and patch-to-beam thickness ratio $t_{p} / t_{b}$ : (a) analytical prediction, and (b) finite element prediction

Assuming negligible longitudinal displacement, the expressions for $U_{\text {conv }}$ and $U_{\mathrm{SC}}^{p}$ for the asymmetric configuration are [11]

$$
\begin{aligned}
U_{\mathrm{conv}} & =\frac{1}{2} \frac{b t_{p}}{s_{11}^{E}} \frac{k_{31}^{2}}{\left(1-k_{31}^{2}\right)}\left(e+\frac{t_{p}}{2}\right)^{2} \frac{1}{(L 2-L 1)}\left[\left.w_{2}^{\prime}\left(x_{1}\right)\right|_{L 1} ^{L 2}\right]^{2} \\
U_{\mathrm{SC}}^{p} & =\frac{1}{2} \frac{b t_{p}}{s_{11}^{E}}\left[\left(e+\frac{t_{p}}{2}\right)^{2}+\frac{1}{1-k_{31}^{2}} \frac{h^{2}}{12}\right] \int_{L 1}^{L 2}\left[w_{2}^{\prime \prime}\left(x_{1}\right)\right]^{2} d x_{1}
\end{aligned}
$$

where $s_{11}^{E}$ is the elastic compliance of the piezopatch under a constant electric field, and $k_{31}$ is the material coupling factor for longitudinal deformation of a thickness polarized piezoelectric rod. Similarly $U_{b}$ can be summed piecewise over the three regions

$$
\begin{aligned}
U_{b}= & \frac{1}{2} I_{b} E_{b} \int_{0}^{L 1}\left[w_{1}^{\prime \prime}\left(x_{1}\right)\right]^{2} d x_{1}+\frac{1}{2} b t_{b} E_{b}\left(\frac{t_{b}^{2}}{12}+\left(e-\frac{t_{b}}{2}\right)^{2}\right) \\
& \times \int_{L 1}^{L 2}\left[w_{2}^{\prime \prime}\left(x_{1}\right)\right]^{2} d x_{1}+\frac{1}{2} I_{b} E_{b} \int_{L 2}^{L 3}\left[w_{3}^{\prime \prime}\left(x_{1}\right)\right]^{2} d x_{1} .
\end{aligned}
$$

Equations (28)-(30) can be specialized for the symmetric case simply by substituting $e=t_{b} / 2$.

For the resonant beams under consideration, the present interest is in maximizing the dynamic electromechanical coupling of the resonant structure. For this purpose, assuming that the mode shapes remain identical for SC and OC electrodes, the effective or dynamic EMCF of a chosen mode of the piezoelectric structure can be related to the OC and SC frequencies $([22,23])$

$$
\mathrm{EMCF}=\left(\frac{U_{\mathrm{OC}}-U_{\mathrm{SC}}}{U_{\mathrm{OC}}}\right)^{1 / 2}=\left(\frac{\omega_{\mathrm{OC}}^{2}-\omega_{\mathrm{SC}}^{2}}{\omega_{\mathrm{OC}}^{2}}\right)^{1 / 2}
$$

where $\omega_{\mathrm{OC}}$ and $\omega_{\mathrm{SC}}$ are the $\mathrm{OC}$ and SC natural frequencies of the chosen mode of the structure. The computation of dynamic EMCF is then performed conveniently using Eq. (31).

\section{Finite Element Analysis}

To verify the analytical model a finite element analysis for both the symmetric and asymmetric configurations was carried out in ANSYS 6.0 [24]. ANSYS element SOLID5 was used in this analysis. SOLID5 is a 20 node three-dimensional linear coupled-field solid element having both piezoelectric and structural properties. It may be noted that the planar isotropy of the piezoelectric material requires the prescription of more material constants than simply the three constants employed in the one-dimensional analytical beam model.

The model is constructed by first defining keypoints, creating volumes, and assigning the material properties to the respective volumes. As in the analytical model, a perfect infinitesimalthickness bond between the actuator and the beam is assumed. The finite element model is also assumed to be lossless. A free three-dimensional (3-D) mesh is constructed that yields a regular brick mesh. All nodes underneath and above the piezopatch(es) 


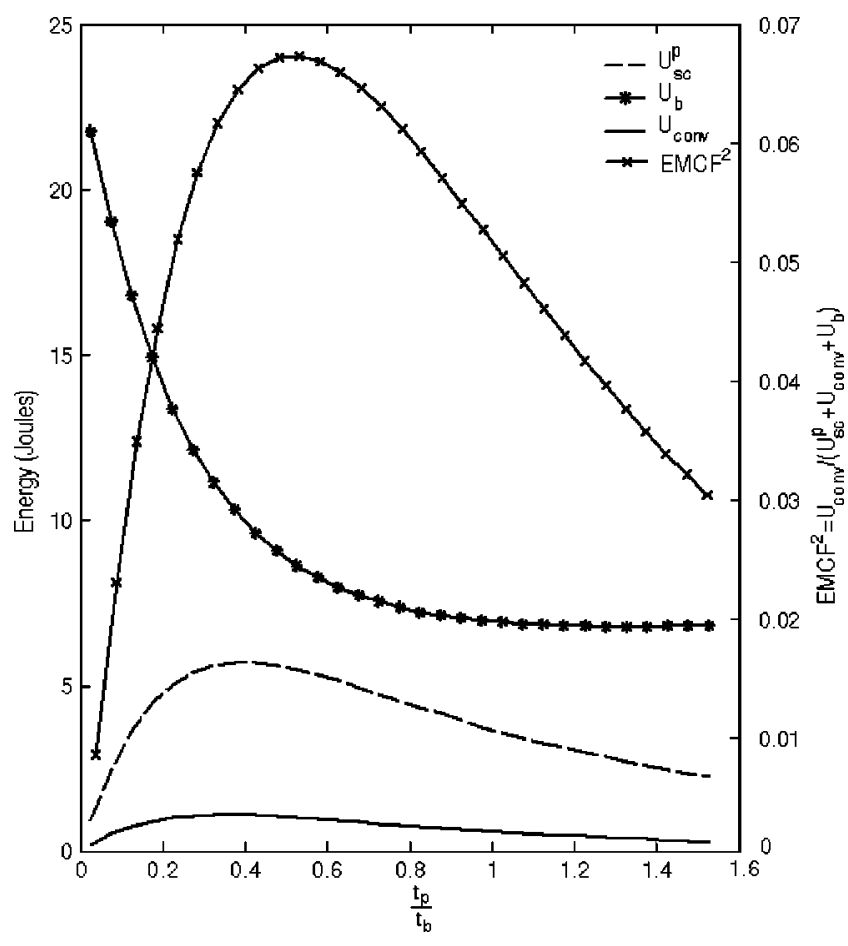

Fig. 6 Variation of $U_{\text {conv }}, U_{\mathrm{SC}}^{p}, U_{b}$ with patch-to-beam thickness ratio $\left(t_{p} / t_{b}\right)$ and the resulting EMCF values according to Eq. (27) for a constant length ratio $(L 2-L 1) / L 3=0.58$. These curves demonstrate why an optimal thickness ratio exists for maximizing the EMCF.

are assigned a voltage coupling for the SC and OC free vibration problem (modal analysis). For the SC condition, the top electrode is assigned zero voltage. For the $\mathrm{OC}$ case, the voltages at all nodes on the top electrode are forced to be identical. Material properties used for the analytical model are presented in Table 1, while the material properties used in the 3-D finite element analysis are presented in matrix form in the following

$$
\begin{gathered}
\mathbf{c}=\left[\begin{array}{cccccc}
6.2 & 3.1 & 3.1 & 0 & 0 & 0 \\
3.1 & 6.2 & 3.1 & 0 & 0 & 0 \\
3.1 & 3.1 & 5.0 & 0 & 0 & 0 \\
0 & 0 & 0 & 1.0 & 0 & 0 \\
0 & 0 & 0 & 0 & 1.0 & 0 \\
0 & 0 & 0 & 0 & 0 & 1.0
\end{array}\right] \times 10^{10} \frac{N}{m^{2}} \\
\mathbf{e}=\left[\begin{array}{ccc}
0 & 0 & -19.84 \\
0 & 0 & -19.84 \\
0 & 0 & 32.5
\end{array}\right] \frac{C}{m^{2}} \\
\epsilon=\left[\begin{array}{ccc}
3.382 & 0 & 0 \\
0 & 3.382 & 0 \\
0 & 0 & 3.382 \\
0 & 0 & 0 \\
0 & 0 & 0 \\
0 & 0 & 0
\end{array}\right] \times 10^{-8} \frac{F}{m}
\end{gathered}
$$

The finite element results thus generated are compared to the analytical results for long and slender beams with different patch lengths. Specifically the modal frequencies (OC and SC) for the asymmetric configuration for typical patch lengths and one thickness $\left(t_{p}=0.178 \mathrm{~mm}\right)$ are presented in Table 2 and are found to be

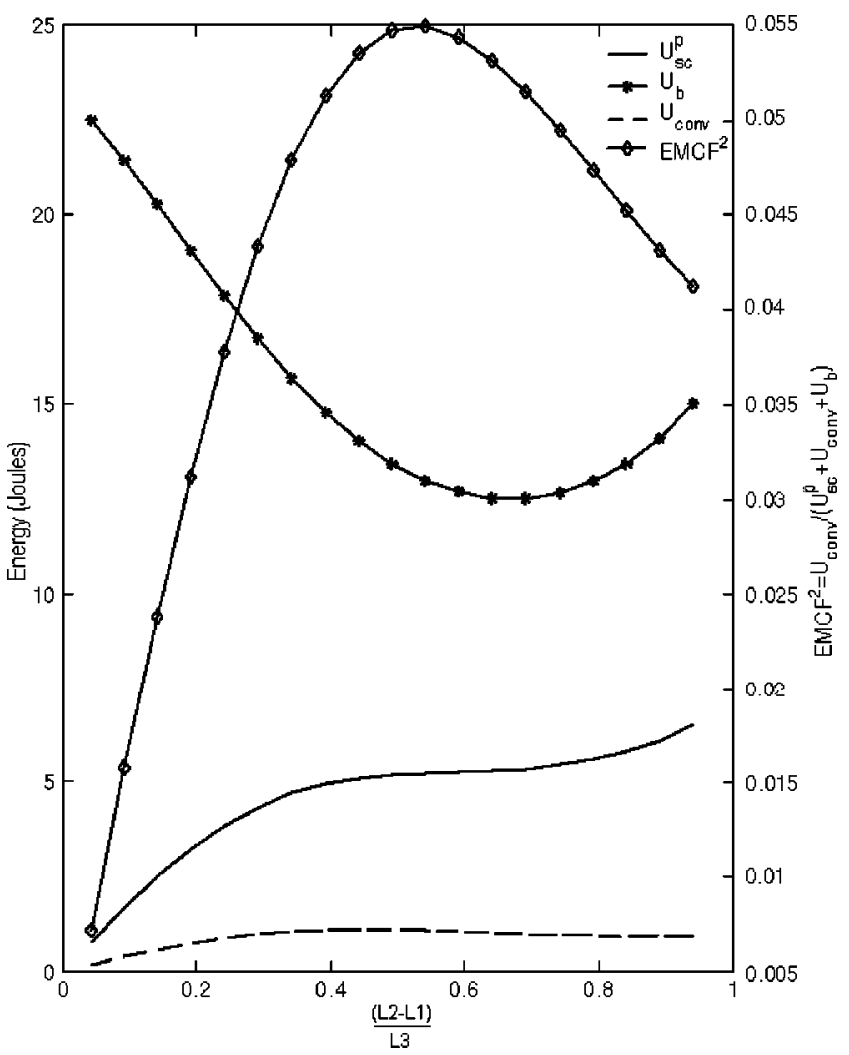

Fig. 7 Variation of $U_{\text {conv }}, U_{\mathrm{SC}}^{p}, U_{b}$ with patch-to-beam length ratio $[(L 2-L 1) / L 3]$ and the resulting EMCF values according to Eq. (27) for a constant thickness ratio $t_{p} / t_{b}=0.5$. These curves demonstrate why an optimal patch-to-beam length ratio exists for maximizing the EMCF.

in close agreement. In Figs. 3 and 4 the predicted mode shapes are compared for the first and second bending modes for both the SC and OC boundary conditions. The length of the patch is set to $L 2-L 1=6.5 \mathrm{~mm}$ while the remaining parameters are listed in Table 1 . The mode shapes predicted by the finite element method are presented in terms of the deflections of the centroidal axis of the beam. Figures $3(a)-3(d)$ demonstrate that the transverse deflections of the analytical and finite element results are in close agreement for the first and the second modes. Figures $4(a)$ and $4(b)$ describe the longitudinal component of the analytical and finite element predictions of the first bending dominated mode for the asymmetric configuration. These two are also in close agreement.

For the symmetric configuration, the finite element and analytical predictions of OC and SC frequencies and mode shapes were also compared and found to be in close agreement. Indeed, the errors were smaller in this case than for the asymmetric configuration, and detailed results are not included here.

\section{Optimization Results}

The main objective of the optimization in this paper is to find the particular geometry of the fan for which the dynamic EMCF of the first bending mode is maximized. For this purpose, the overall fan length, $L 3$, the distance from the clamp to the beginning of the patch, $L 1$, and the beam thickness, $t_{b}$, are kept constant and the thickness of the patch, $t_{p}$, and the distance from the clamp to the end of the patch, $L 2$, are varied as described in Table 1. In Fig. 5, EMCF contour plots are provided for both the analytical and the finite element models for the asymmetric configuration. The optimal geometries predicted by these plots are in close agreement with each other.

Several important observations can be made from these plots: 

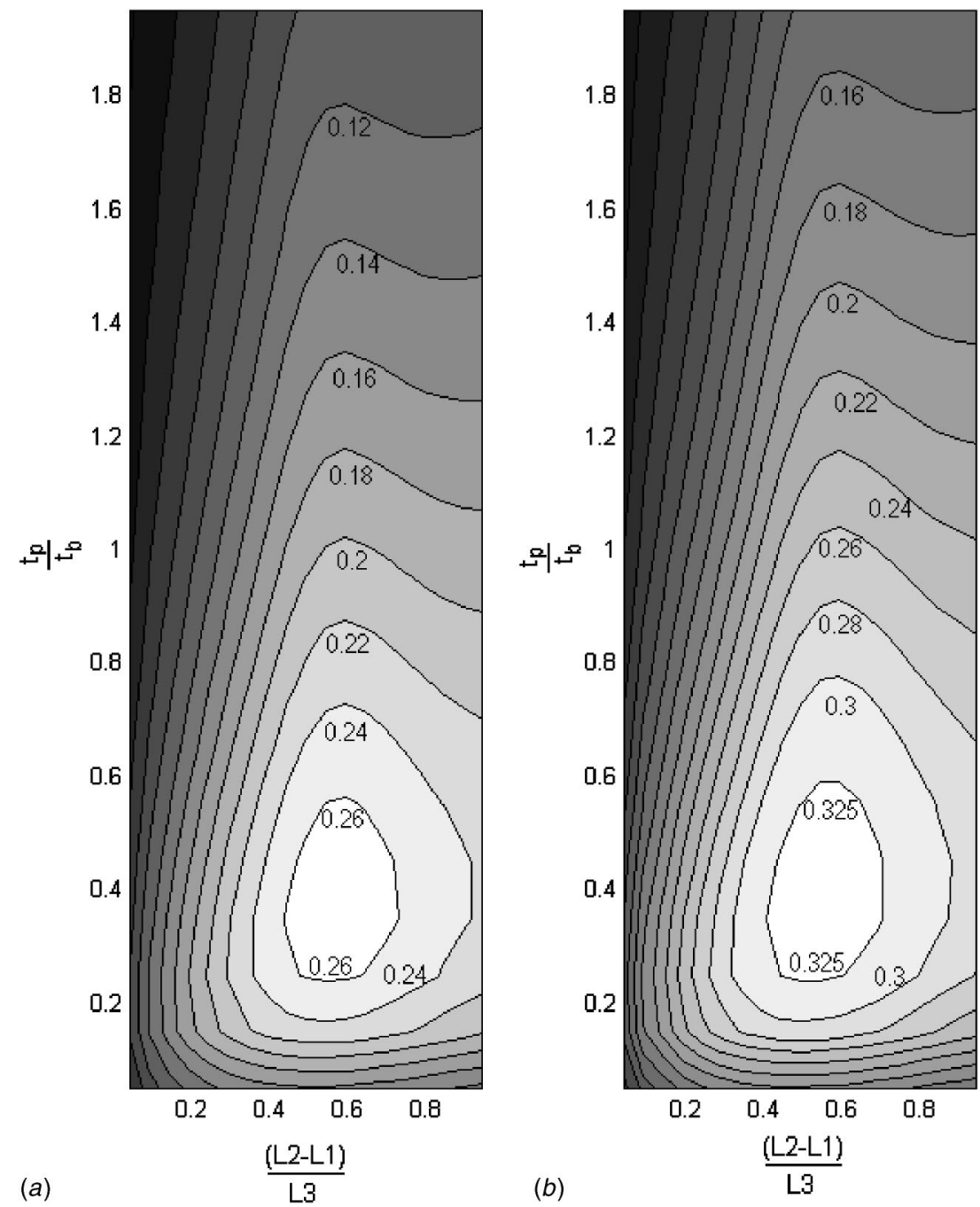

Fig. 8 EMCF variation of the first bending mode of a symmetric resonator with patch-to-beam length ratio $(L 2-L 1) / L 3$ and patch-to-beam thickness ratio $t_{p} / t_{b}$ : (a) analytical prediction, and (b) finite element prediction

1. There is a clear optimum length ratio, $(L 2-L 1) / L 3$, and thickness ratio $t_{p} / t_{b}$ for which the dynamic EMCF of the first bending mode is maximized. This optimal geometry corresponds to $t_{p} / t_{b} \approx 0.5,(L 2-L 1) / L 3 \approx 0.6$. However, while the optimal fan geometry is identical using the theoretical and finite element analysis, the EMCF contour values are not identical.

2. It may be noted that these contour plots are specific to the chosen piezoelectric and beam material properties. The optimal geometry varies depending on these properties.

The existence of an optimum in the EMCF plots can also be interpreted using Eqs. (27)-(30). In Fig. 6 the energies, $U_{\mathrm{SC}}^{p}, U_{b}$, $U_{\text {conv }}$ are plotted for a constant length ratio $[(L 2-L 1) / L 3$ $=0.58]$ using Eqs. (28), (29), and (30) and analytical mode shapes. Also plotted are the resulting EMCF values using Eq. (27). In Fig. 7 these quantities are plotted for a constant thickness ratio $\left(t_{p} / t_{b}=0.5\right)$. When the thickness ratio is varied with a constant length ratio (Fig. 6), $U_{\mathrm{SC}}^{p}$ and $U_{\text {conv }}$ have local maxima near $t_{p} / t_{b}=0.4$. $U_{b}$ however decreases with increasing thickness because the piezopatch stiffens thus reducing the beam strain energy. When the thickness ratio is kept constant and the length ratio is varied (Fig. 7), there are two competing factors that come into play in Eqs. (28)-(30). As the length ratio is increased, the overall structure stiffens resulting in less deflection and strain energy in the beam. Thus initially $U_{b}$ decreases. But with increasing length ratio, the second integral in Eq. (30) also increases relative to the third integral, and $U_{b}$ increases beyond $(L 2-L 1) / L 3 \geqslant 0.7$. For these reasons the EMCF is maximized for specific thickness and length ratios.

Similar observations can be made for the symmetric configuration (Fig. 8). In this case, the optimal length ratio [( $L 2$ $-L 1) / L 3 \approx 0.6]$ remains the same, but the optimal thickness ratio $\left(t_{p} / t_{b} \approx 0.4\right)$ is slightly lower than the optimal asymmetric configuration. Thus the optimal geometry for the symmetric resonator requires more piezoelectric material than the optimal geometry of the asymmetric resonator. Again, like the asymmetric configuration, EMCF contour values are higher for the finite element model compared to the analytical model. The optimal geometries will depend on the chosen material properties of the beam and the piezopatch.

\section{Experimental Verification of Optimization Results}

5.1 Experimental Setup. To verify the theoretical and finite element results, experiments are performed on an asymmetrically configured piezoelectric fan-like structure consisting of a piezopatch attached to a thin steel blade with superglue. The initial dimensions of the steel blade and piezopatch are shown in Table 3. The effect of patch to beam length ratio on the EMCF of the first bending mode are measured experimentally for this system. 
Table 3 Properties and dimensions of the piezoceramic and the steel blade used in the experiment. Piezoelectric material properties are obtained from Piezo Systems, Inc. catalog 3.

\begin{tabular}{lll}
\hline \hline & & \multicolumn{1}{c}{ Physical quantity } \\
\hline$E_{b}$ & Young's modulus of the beam (steel) & $21 \times 10^{10} \mathrm{~N} / \mathrm{m}^{2}$ \\
$E_{p}$ & Young's modulus of the actuator (PSI-5A-S4-ENH) & $6.6 \times 10^{10} \mathrm{~N} / \mathrm{m}^{2}$ \\
$\rho_{b}$ & Density of the beam (steel) & $7850 \mathrm{~kg} / \mathrm{m}^{3}$ \\
$\rho_{p}$ & Density of the actuator (PSI-5A-S4-ENH) & $7800 \mathrm{~kg} / \mathrm{m}^{3}$ \\
$t_{b}$ & Thickness of the beam & $0.1016 \mathrm{~mm}$ \\
$t_{p}$ & Thickness of the actuator & $0.1905 \mathrm{~mm}$ \\
$b$ & Width of beam and actuator & $10 \mathrm{~mm}$ \\
$L 1$ & Length of region 1 & $2.8 \mathrm{~mm}$ \\
$L 2-L 1$ & Length of patch & $10.8 \mathrm{~mm}$ \\
$L 3$ & Overall length of the structure $L 3$ & $51.65-25.65 \mathrm{~mm}$ \\
$K_{33}$ & Relative dielectric constant & 1800 \\
$\epsilon_{0}$ & Permittivity of free space & $8.8542 \times 10^{-12} \mathrm{C} / \mathrm{V} \mathrm{m}$ \\
$d_{31}$ & Piezoelectric strain coefficient & $-190 \times 10^{-12} \mathrm{~m} / \mathrm{V}$ \\
\hline \hline
\end{tabular}

To investigate this, $L 1$ and $L 2$ are maintained constant while $L 3$ is decreased gradually in steps. This process was chosen because the reduction in $L 3$ can be achieved without inducing errors from clamping and unclamping of the structure.

5.2 Impedance Measurement. At each $L 3$, the SC and OC frequencies of the structure are obtained from impedance plots generated by the Impedance Analyzer (HP/Agilent 4294A). Figure 9 shows the experimental setup. Figure 10 represents a typical impedance plot near the first resonance frequency of the structure together with the corresponding finite element and analytical frequencies based on the material properties listed in Table 3. For a lossless resonator, the frequency at every local minimum of the impedance plot represents a natural frequency. Thus the first local minimum indicates the first SC frequency or the resonant frequency. Similarly, for a lossless resonator, the corresponding local maximum is the first antiresonance or the OC frequency of the structure.

It may be noted that the SC and OC frequencies predicted by the finite element and the analytical models are slightly lower than the corresponding experimental values. This discrepancy is likely due to the lack of inclusion of the bonding layer mechanics into the models. To demonstrate this, finite element calculations in ANSYS were performed on a resonator with a thin elastic bonding layer of low modulus $\left(10 \mathrm{GPa}\right.$ ) and density $1100 \mathrm{~kg} / \mathrm{m}^{3}$ (that of ethyl cyano-acrylate, the major ingredient of the bonding used in

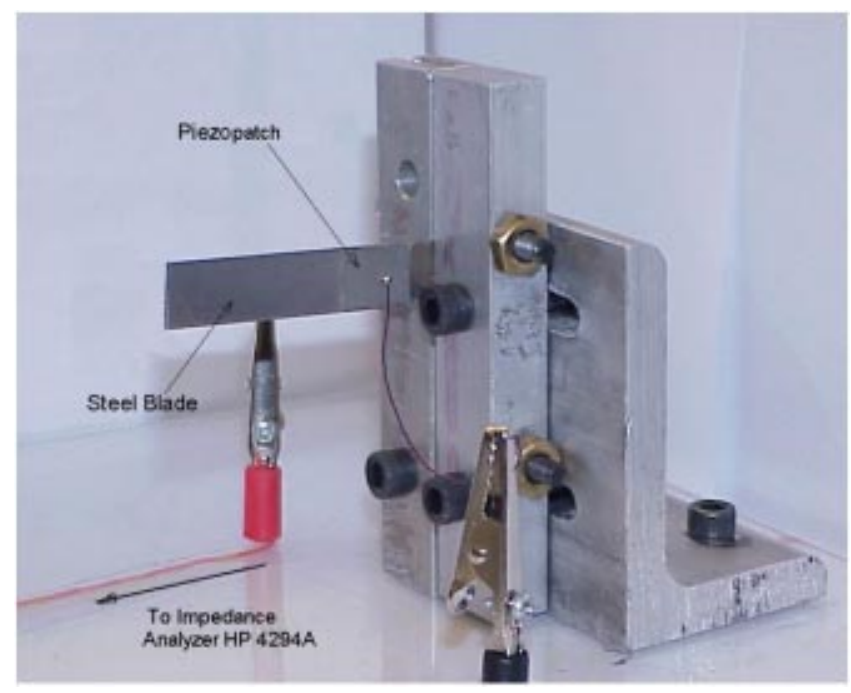

Fig. 9 Experimental setup for the measurement of EMCF with varying blade lengths of an asymmetrically configured flexural resonator the experiment). As shown in Fig. 10, inclusion of the bonding layer results in an increase in both the SC and the OC frequencies and they approach the experimental frequencies.

5.3 Optimization Results and Discussion. At each L3, the impedance data are collected ten times and their average and $\pm 1 \sigma$ deviations computed. The results are shown in Fig. 11, along with analytical and finite element predictions based on the methods described earlier. Material properties of the finite element and the analytical models are listed in Table 3. Figure 11 shows clearly that the experimental EMCF reaches a local maximum at $(L 2-L 1) / L 3 \approx 0.32$ which is close to the analytical and finite element predictions of 0.35 and 0.33 respectively.

The apparent underprediction of the measured EMCF values by the finite element and the analytical EMCF calculations can be explained from [23] and [25]. Specifically, the measured EMCF and $\mathrm{OC}$ and SC frequencies are based on the minima and the

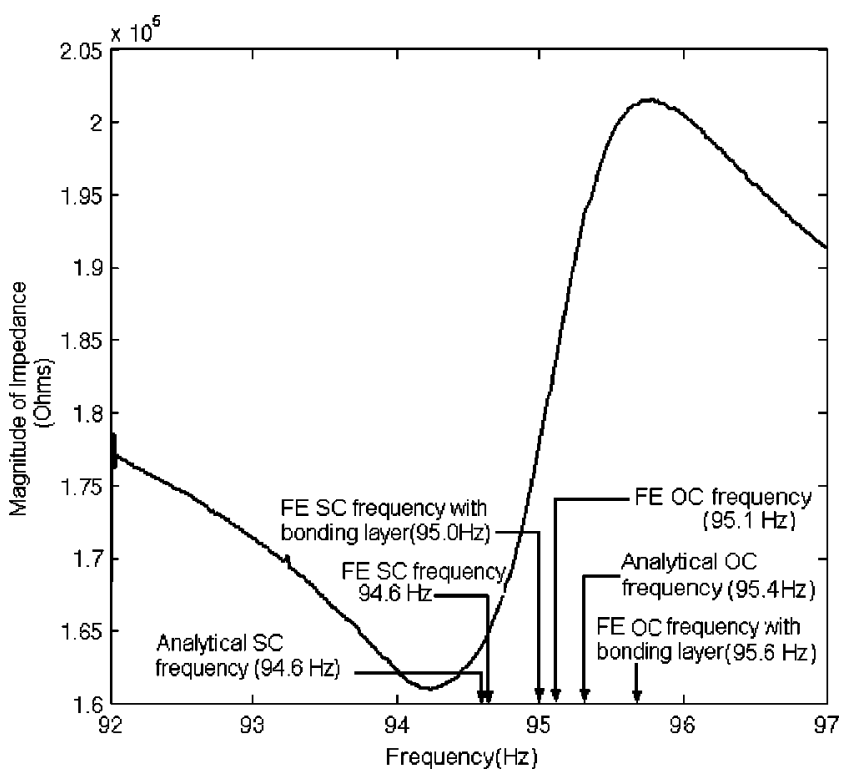

Fig. 10 A typical impedance plot with steel blade of width 10 $\mathrm{mm}$, height $0.1016 \mathrm{~mm}$, and length $36.65 \mathrm{~mm}$. The piezopatch used is PSI-5A-S4-ENH (thickness $=0.1905 \mathrm{~mm}, \mathrm{Ep}=66 \mathrm{GPa}$ ) and of length $(L 2-L 1)=10.8 \mathrm{~mm}$ and $L 1=2.3 \mathrm{~mm}$. For a lossless resonator, the minimum in the impedance plot represents the SC frequency or the series resonance frequency, whereas the maximum represents the $\mathrm{OC}$ frequency or the parallel resonance frequency. Corresponding $\mathrm{SC}$ and $\mathrm{OC}$ frequencies for the analytical, finite element model with and without a thin bonding layer are also provided for comparison. 


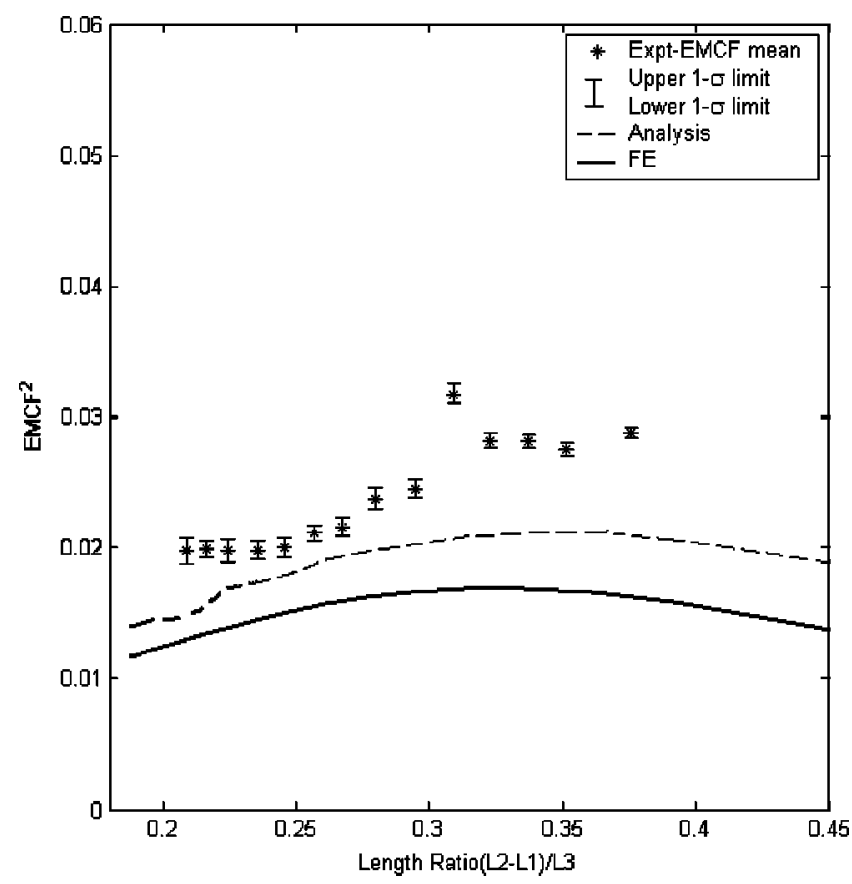

Fig. 11 Variation with length ratio of EMCF of the first bending mode of the asymmetric resonator. Experimental, analytical, and finite element predictions are shown.

maxima of the measured impedance. This, however, is not accurate in the presence of unmodeled losses. In fact it can be shown [22] that the difference between the actual SC and OC frequencies is less than the difference in frequencies corresponding to maxima and minima of the measured impedance plots. This implies that the actual experimental EMCF is lower than the measured experimental EMCF.

\section{Conclusions}

This paper investigates the dynamic response of resonators consisting of symmetrically or asymmetrically configured piezopatches bonded on thin elastic beams. An analytical model is developed for both configurations using thin composite beam theory and through the use of Hamilton's principle. The analysis predicts that transverse and longitudinal motions are coupled in the asymmetric configuration, but they decouple for the symmetric problem. The analytical solution is found to match closely with the finite element predictions for both the symmetric and the asymmetric configurations.

The analytical predictions are used to investigate optimal patchto-beam length and thickness ratios to maximize electromechanical coupling factor (EMCF) of such structures. It is shown that depending on the configuration (symmetric or asymmetric) and the choice of materials, specific length and thickness ratios maximize the EMCF for certain bending-dominated modes of these structures. Experiments are performed to investigate the variation with length ratio of the EMCF of the first bending mode of an asymmetrically configured resonator. The results indicate that the analytical and finite element predictions of the optimal length ratio match those found in the experiments. The analytical and finite element EMCF predictions underpredict somewhat the measured EMCF values. This discrepancy is likely due to the inherent losses in the experiment.
The results indicate that the analytical model is useful for the optimization of the geometry of such resonators. The results are important for the design of piezoelectric fans, optical beam choppers, ultrasonic choppers, MEMS density sensors, resonating viscometers, MEMS resonating acclerometers, ultrasonic motors, automatic tooth brushes, and dentistry tools.

\section{Acknowledgments}

The authors acknowledge the financial support for this work from the Cooling Technologies Research Center (CTRC) at Purdue University. The authors would also like to thank Dr. Ing. Kai Wolf of Elliptec AG for valuable discussions on electromechanical coupling factor.

\section{References}

[1] Toda, M., 1979, "Theory of air flow generation by a resonant type PVF2 bimorph cantilever vibrator," Ferroelectrics, 22, pp. 911-918.

[2] Yoo, J. H., Hong, J. I., and Cao, W., 2000, "Piezoelectric ceramic bimorph coupled to thin metal plate as cooling fan for electronic cooling," Sens. Actuators, A, 79, pp. 8-12.

[3] Campbell Jr., J. S., Glezer, W. Z., and Hartley, J. G., 1998, "Thermal management of a laptop with synthetic air microjets," IEEE Intersociety Conference on Thermal Phenomena, pp. 43-50.

[4] Crawley, E. F., and de Luis, J., 1987, "Use of piezoelectric actuators as elements of intelligent structures," AIAA J., 25, pp. 1373-1385.

[5] Crawley, E. F., and Anderson, E. H., 1990, "Detailed model of piezoceramic actuation of beams," J. Intell. Mater. Syst. Struct., 1, pp. 5-25.

[6] Main, J. A., Garcia, E., and Howard, D., 1994, "Optimal placement and sizing of paired piezoactuators in beams and plates," Smart Mater. Struct., 3, pp. 373-381.

[7] Abramovich, H., 1998, "Deflection control of laminated composite beams with piezoceramic layers-closed form solutions," Compos. Struct., 43, pp. $217-231$.

[8] Gibbs, G. P., and Fuller, C. R., 1992, "Excitation of thin beams using asymetric piezoelectric actuators," J. Acoust. Soc. Am., 6, pp. 3221-3227.

[9] Pan, X., and Hansen, C. H., 1994, "Piezoelectric crystal vs point force excitation of beams and plates," J. Intell. Mater. Syst. Struct., 5, pp. 363-370.

[10] Brennan, M. J., Elliot, S. J., and Pinnington, R. J., 1995, "The dynamic coupling between piezoceramic actuators and a beam,” J. Acoust. Soc. Am., 102, pp. 1931-1942.

[11] Wolf, K.-D., 2000, "Electromechanical energy conversion in asymmetric piezoelectric bending actuators," Ph.D. thesis, Technische Universität Darmstadt, Germany.

[12] Pan, J., Hansen, C. H., and Snyder, S. D., 1992, "A study of the response of a simply supported beam to excitation by a piezoelectric actuator," J. Intell. Mater. Syst. Struct., 3, pp. 3-16.

[13] Rivory, J. F., Hansen, C. H., and Pan, J., 1994, "Further studies of the dynamic response of a simply supported beam excited by a pair of out-of-phase piezoelectric actuators," J. Intell. Mater. Syst. Struct., 5, pp. 654-663.

[14] Kim, S. J., and Jones, J. D., 1996, "Influence of piezo-actuator thickness on the active vibration control of a cantilever beam,' J. Intell. Mater. Syst. Struct., 6, pp. 610-623.

[15] Rontong, J. A., Wright, J. R., Wynne, R. J., and Tomlinson, G. R., 1997 "Modeling of a hybrid constrained layer/piecewise approach to active damping," ASME J. Vibr. Acoust., 119, pp. 120-130.

[16] Lobontiu, N., Goldfarb, M., and Garcia, E., 1999, “Achieving maximum tip deflection during resonant excitation of piezoelectrically actuated beams," J. Intell. Mater. Syst. Struct., 10, pp. 900-913.

[17] Barboni, R., Mannini, A., Fantini, E., and Gaudenzi, P., 2000, "Optimal placement of PZT actuators for the control of beam dynamics," Smart Mater. Struct., 9, pp. 110-120.

[18] Wang, Q., and Wang, C. M., 2000, “Optimal placement and size of piezoelectric patches on beams from the controllability perspective," Smart Mater Struct., 9, pp. 558-567.

[19] Bürmann, P., Raman, A., and Garimella, S. V., 2002, "Dynamics and topology optimization of piezoelectric fans," IEEE Trans. Compon., Packag. Manuf. Technol., Part A, 25, pp. 592-600.

[20] Tiersten, H. F., 1969, Linear Piezoelectric Plate Vibrations, Plenum, New York.

[21] Waterloo Maple, Inc., 2002, Academic (ACD) Maple 8 student edition for Windows, Waterloo, Ontario, Canada

[22] Berlincourt, D. A., Corran, D. R., and Jaffe, H., 1964, "Piezoelectric and piezomagnetic materials and their function in transducers," Phys. Acoust., 1A, pp. $169-270$.

[23] Hunt, F. V., 1954, Electroacoustics, Wiley, New York.

[24] ANSYS Inc., 2001, ANSYS 6.0 Documentation.

[25] IRE Standards on Piezoelectric Crystals, 1961, Proc. IRE, 49, pp. 1162-1169. 